\title{
A Least Squares Database Approach for SAR Interferometric Data
}

\author{
Stefania Usai
}

\begin{abstract}
This paper presents a least squares (LS) approach for the retrieval of a temporal deformation sequence from a set of interferometric synthetic aperture radar images. The method uses a database of interferograms spanning different long- and short-term intervals, and by solving all the deformations as a unique LS problem provides a chronologically ordered sequence, i.e., a picture of the development of the deformation pattern in time. The approach is illustrated in detail and discussed with respect to both the results of its application on a case study and to possible alternative methods.
\end{abstract}

Index Terms-Ground displacements, least squares methods, synthetic aperture radar (SAR) interferometry.

\section{INTRODUCTION}

D ESPITE being a relatively new technique, differential synthetic aperture radar (SAR) interferometry (D-InSAR) [1], [2] is already widely used for the measurement of deformations. There are several examples of its application for the measurement and modeling of fast displacements such as those due to earthquakes and volcanic events [3], [4]. In the case of slow deformations, however, the efficacy of this technique is seriously limited by temporal decorrelation [5], [6]: interferometric combinations (also called interferograms) on intervals of more years are in most cases degraded to such a point that it is practically impossible to retrieve unambiguous information from them. The alternative solution for long-term application is then to consider, instead of one single interferogram, a series of shorter term ones and sum them up to span the desired interval. Because a particular area is not necessarily imaged whenever it is visible, and because baseline limitations do not always allow to obtain the desired interferometric combinations, it is in general rather difficult to obtain interferograms on consecutive time intervals. In fact, in most cases the generated interferograms do extend over partially overlapping time intervals. The problem then arises how to order the information from all these intervals to give a deformation history.

This paper describes a method to solve this problem, and more in general to enhance the feasibility of SAR interferometry for deformation studies. In this least squares (LS)-based approach, a set of interferometric images is merged to give a time series describing the temporal evolution of the deformations. The method, by using the redundancy of information contained in temporally overlapping interferograms, additionally allows

Manuscript received March 11, 2002; revised October 14, 2002

The author is with the Delft Institute for Earth-Oriented Space Research (DEOS), Delft University of Technology, Delft 2629 HS, The Netherlands (e-mail: Stefania.Usai@1r.tudelft.nl).

Digital Object Identifier 10.1109/TGRS.2003.810675 to reduce the effects of both processing errors and decorrelation noise. Finally, because it allows a dense temporal sampling of the displacements, it constitutes a new tool for their geophysical modeling and study.

The paper is organized as follows. After a description of the procedure followed to generate an interferometric database in Section II, the LS approach is explained in Section III. Section IV illustrates the results of the application of this method in the Phlegrean Fields area, while Section V is devoted to the discussion of the approach, not only with respect to the results of the case study but also in the more general context of the current research in SAR Interferometry and of its applications. Finally, conclusions on the presented research and recommendations for future work are given in Section VI.

\section{INTERFEROMETRIC AND POSTINTERFEROMETRIC PROCESSING}

The proposed approach can be applied on a generic set of interferometric deformation images of the same area. With the term "interferometric deformation images" it is intended that the interferograms have been unwrapped, the topographic component of the signal subtracted, and that the unwrapped phase values are referred to the same zero deformation point and converted in deformations values along the line-of-sight direction. The LS procedure is, therefore, independent from the interferometric processing itself, and in this sense it can be interpreted as an interferometric postprocessing step. Nonetheless, when creating a database for the purpose of applying this approach, it is advisable to follow a slightly adapted procedure for the interferometric processing. The procedure, described in detail in [7], consists basically in coregistering all the SAR images to a same database master prior to interferogram formation. The advantage is that the resulting interferograms are all matching among each other at the subpixel level, which allows to merge them directly in the form of unwrapped absolute phase values (converted in centimeters, if desired). In this way, there is no need to apply geocoding, a processing step which constitutes an additional error source for input data to the LS adjustment.

The input to the LS procedure is thus a set of such interferometric deformation images, all matching at the subpixel level and showing the absolute deformation, in phase or centimeter units, along the satellite line-of-sight direction.

As a first step, the correctness of the absolute deformations is checked by iteratively computing linear combinations of interferograms [7]. The phase value chosen as reference in a certain interferogram could in fact be biased, causing a shift of the whole deformation pattern. Interferogram-related biases are 


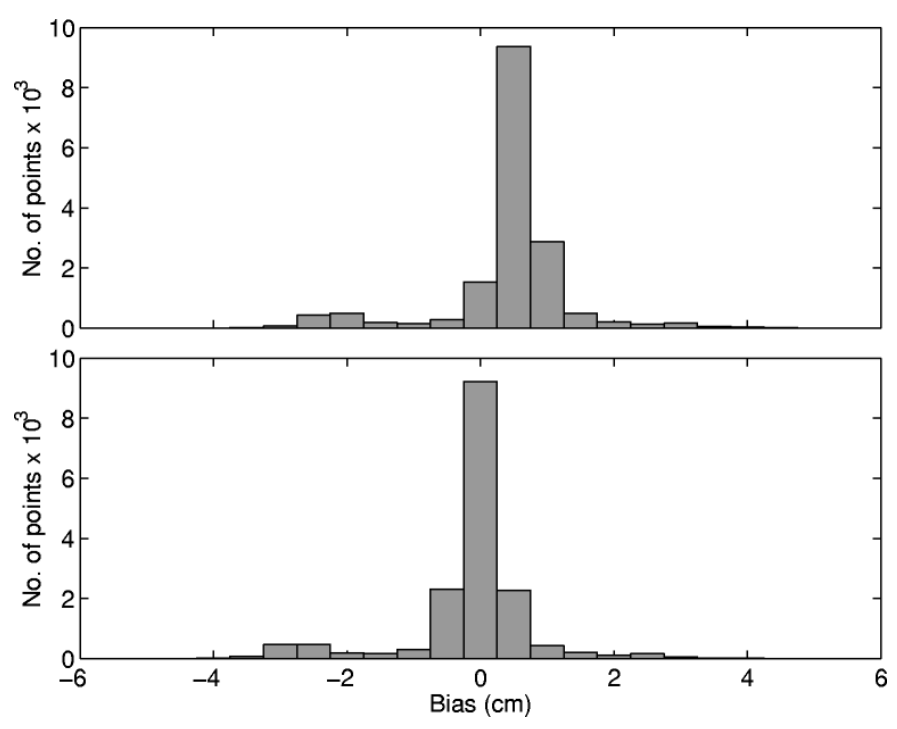

Fig. 1. (Top) Histogram of a linear combination of three interferograms forming a closed loop. On $x$ axis are represented the deformations in centimeters. The maximum is clearly shifted with respect to zero, indicating the presence of a bias. (Bottom) Same histogram after the correction for a bias, which in this case was estimated at $0.5 \mathrm{~cm}$.

mainly caused by phase-unwrapping errors, due to the presence of decorrelation noise between the reference point or area and other parts of the imaged scene. They can be detected and at the same time estimated by combining the deformation maps forming what can be called a "closed loop" in time.

Supposing to have three interferograms $\mathcal{A B}, \mathcal{B C}$, and $\mathcal{C} \mathcal{A}$ taken at subsequent days $\mathcal{A}, \mathcal{B}$, and $\mathcal{C}$, the linear combination $\mathcal{A B}+\mathcal{B C}+\mathcal{C} \mathcal{A}$ of the absolute deformation values for each pixel in the three interferograms should give in principle as a result zero

$$
D_{\mathcal{A B}}(i, j)+D_{\mathcal{B C}}(i, j)+D_{\mathcal{C A}}(i, j)=0
$$

where $D$ is the absolute deformation expressed in length units (centimeters), and $(i, j)$ are the coordinates of a certain pixel.

Due to the presence of noise in the three interferograms, a noise caused by all different effects and which can be assumed to be random, the linear combination of the deformation values will not be overall zero. An histogram of its values, however, should show anyway a clear maximum around the zero value, as for example in the upper plot of Fig. 1. It is intuitive that if one of the interferograms is biased, for example if

$$
D_{\mathcal{A B}}^{\prime}(i, j)=D_{\mathcal{A B}}(i, j)+K, \quad K=\text { const } .
$$

then (1) will take the form

$$
D_{\mathcal{A B}}^{\prime}(i, j)+D_{\mathcal{B C}}(i, j)+D_{\mathcal{C} \mathcal{A}}(i, j)=K
$$

which means also that the histogram of the values of the linear combination will be centered in $K$, as depicted on the lower plot of Fig. 1. One can, therefore, check for the presence of a bias by considering an histogram of a linear combination of interferograms, and take the value at its maximum as an estimate of this bias. Notice that to determine the exact value of the maximum, it is necessary to compute the distribution a certain number of times, shifting each time the bin edges.
Of course, assessing which one among $\mathcal{A B}, \mathcal{B C}$, and $\mathcal{C} \mathcal{A}$ has to be corrected is not always straightforward. Most of the times this can be determined by comparing each of the three interferograms with more other interferograms, forming other "closed loops": it can be expected that a biased interferogram will cause biases in all the loops where it is considered and can therefore be identified quite easily. Moreover, knowing the expected deformation in the area, for example from other survey methodologies, can help to identify the interferograms giving plausible deformation values. These can thus be taken as the "good" ones and considered as a starting set of reliable interferograms to which all the others can be compared with the histogram method. Eventually, if the biases are estimated and subtracted correctly, the interferograms should be consistent as a whole set. This can be assessed again by checking that all the possible closed loop combinations of the interferograms give an histogram centered on zero.

\section{LS SOLUTION COMPUTATION AND TESTING}

The input for the LS adjustment is a set $y=\left[I_{1}, \ldots, I_{N}\right]$ of $N$ interferometric deformation maps, generated from $M$ SAR images taken at the days $x=\left[d_{1}, \ldots, d_{M}\right]$. The day $d_{1}$ corresponding to the first image is taken as reference and the deformations at each of the other $(M-1)$ days relative to this day are found as solutions of the problem [7]-[9]

$$
y=A x \quad Q_{y}=\sigma^{2} Q
$$

with $y$ and $x$ given above, where in $x$ the element $d_{1}$ has not been considered. In (4), $Q_{y}$ is the a priori covariance matrix of the observations; $\sigma^{2}$ is the variance and $Q$ the cofactor matrix. Since an unweighted LS approach is used, $Q_{y}$ (and thus $Q$ ) is assumed to be the unit matrix, and $\sigma^{2}=1$.

In the system matrix $A$, each row corresponds to an interferogram, while the columns correspond to the days. For interferogram $I_{k}=I_{d_{i}}-I_{d_{j}}$ the values (on row $k$ ) are all zero except at columns $i$ and $j$, where they are respectively +1 and -1 , the sign being determined by the interferometric difference.

The unweighted LS solution $\hat{x}$ of (4) is straightforward

$$
\begin{aligned}
\hat{x} & =\left(A^{t} A\right)^{-1} A^{t} y \\
Q_{\hat{x}} & =\left(A^{t} A\right)^{-1} .
\end{aligned}
$$

In order to determine the quality of the solution, an overall model test is applied during the LS computation. The overall model test of a dataset gives a measure of the discrepancy between data and used model and is defined as [10]

$$
T_{N-M}=\frac{\hat{e}^{T} Q^{-1} \hat{e}}{(N-M) \sigma^{2}}
$$

with $\hat{e}$ the least squares residual vector. In the interferometric case it can be reasonably assumed that, in absence of systematic errors, the residuals relative to the set of values assumed by the same pixel in all the interferograms are normally distributed. In this case the test statistic has a Fisher distribution with degrees of freedom $N-M$ and $\infty$. For each pixel, the determined solution was tested against such a distribution within a $95 \%$ confidence level. As it will be shown also in Section IV for the considered 
case study, failing of this test can be considered as an indicator of systematic effects, which invalidate the basic assumption of a normal distribution of the residuals.

\section{A. LS Solution Update via Recursive Estimation}

One of the drawback of LS methods is that, in general, the solution has to be recomputed whenever new data are added to the existing database. This is however not necessary if, as in the interferometric case, the data can be assumed to be uncorrelated.

Suppose $y_{1}$ represents the data used for the computation of the most recent solution, say with the standard unweighted LS procedure of (5) and (6)

$$
\begin{aligned}
\hat{x}_{1} & =\left(A_{1}^{t} A_{1}\right)^{-1} A_{1}^{t} y \\
Q_{\hat{x}_{1}} & =\left(A_{1}^{t} A_{1}\right)^{-1} .
\end{aligned}
$$

Assuming that a newly acquired set of data $y_{2}$ with corresponding covariance matrix $Q_{2}$ has to be added to the solution, the new LS problem to be solved is now

$$
\left(\begin{array}{l}
y_{1} \\
y_{2}
\end{array}\right)=\left(\begin{array}{l}
A_{1} \\
A_{2}
\end{array}\right) x
$$

The new (updated) estimate of $x$ is then given, in the unweighted LS case $\left(Q_{1}=Q_{2}=I\right)$, by [11]

$$
\hat{x}_{2}=\hat{x}_{1}+Q_{\hat{x}_{1}} A_{2}^{t}\left(I+A_{2} Q_{\hat{x}_{1}} A_{2}^{t}\right)^{-1}\left(y_{2}-A_{2} \hat{x}_{1}\right)
$$

and the associated variance is

$$
Q_{\hat{x}_{2}}=Q_{\hat{x}_{1}}-Q_{\hat{x}_{1}} A_{2}^{t}\left(I+A_{2} Q_{\hat{x}_{1}} A_{2}^{t}\right)^{-1} A_{2} Q_{\hat{x}_{1}} .
$$

\section{B. Addition of Data by Linear Interpolation}

When one or more interferograms are added to the dataset, they can be used to update the solution only if they can be connected to the existing set of interferograms. In other words, at least one of a set of interferograms formed with newly acquired images has to be a combination with a preexisting image, in order to give a unique solution. This has to be true for each disjoint subset added; otherwise the system matrix $A$ becomes a block diagonal matrix, and the normal matrix $\left(A^{t} A\right)$ is singular. The necessity of having always at least one joint interferogram is not such a strong limitation as it could appear at a first sight. It is often possible to form at least one interferogram by combining one newly acquired image with the preexisting ones in the database, especially if this is already rather large. If, however, no such interferogram can be generated, a "simulated" deformation image can be generated from the preexisting solution and inserted together with the new subset to compute the updated solution. The simulated deformation image is computed, at one of the dates of the subset to be added, by linear interpolation of the solution at its nearest dates of the preexisting set.

The subset is joined to the preexisting set by interpolating only at one date. Joining the subset by interpolation at all the dates of the new dataset would also be possible, but it would lead to the necessity of introducing weighting. In this case, in fact, the (different) length of the time interval considered for the interpolation at each date should be taken into account. Also, the distance of the estimated point to the limits of the interval should

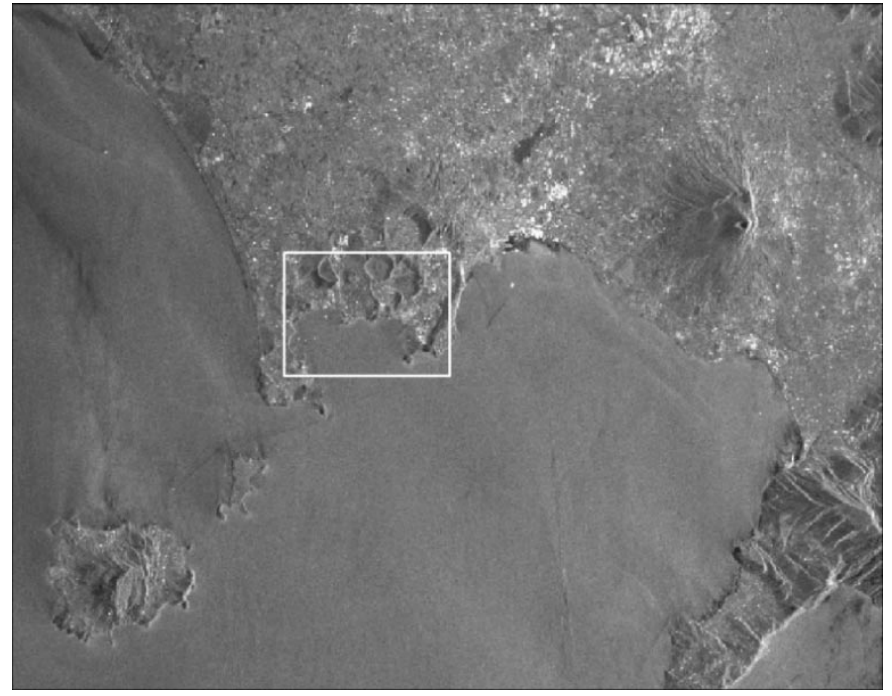

Fig. 2. European Remote Sensing (ERS) satellite amplitude image of the Neapolitan area (Italy). The Vesuvius volcano is recognizable on the right side of the image. The inset box indicates the area of the Phlegrean Fields considered for the computation of the LS solution.

be considered in weighting, as the reliability of the estimation increases passing from the center of the interval to one of its extremes.

Of course, connecting two disjoint sets in this way means assuming a linear trend of the deformations within the time interval considered for the interpolation, an assumption whose validity is dependent on the terrain deformation history and on the length of the time interval itself. For this reason, therefore, it is advisable to choose always the shortest interval for the interpolation, and connect the new subset to the existing one where the temporal sampling is densest.

Finally, it has also to be pointed out that addition by interpolation might be necessary only temporarily: as newer images are added to the dataset in fact, the chance to be able to connect directly two previously disjoint sets increases.

\section{Phlegrean Fields Test Case}

The proposed approach has been applied to measure the terrain displacements in the period 1993-1999 at the Phlegrean Fields (Naples, Italy). This densely populated, highly hazardous area in the Vesuvius volcanic complex is known to be subject to alternating phases of uplift and subsidence. After two major crisis in the years 1969-1972 and 1982-1984, which caused a total maximum uplift of about $3.5 \mathrm{~m}$ [12], this constantly monitored area is in the course of the last years mostly subsiding, at a rate of a few millimeters per month. However, short-term uplift episodes still occur, accompanied by seismic events: the last deformation inversion took place between March and July 2000 , with a total maximum uplift of $4 \mathrm{~cm}$ [13]. A SAR intensity image of the Phlegrean Fields is shown in Fig. 2. The set of 20 ERS-1 and ERS-2 SAR images considered for this study is listed in Table I. The images are labeled in chronological order, with "day numbers": considering the deformation rate, Tandem images have been assumed to contain the same deformation information and therefore correspond to the same day. Fig. 3 
TABLE I

ERS SCENES OF THE PHLEGREAN FIELDS CONSIDERED FOR THE GENERATION of the Dataset. The Scenes All Have Frame No. 2781. The Fourth Column SHOWS THE Day Number, I.E., THE SERIAL Number, Assigned to the IMAge (SEe Also TeXt)

\begin{tabular}{c|c|c|c}
\hline ERS1/2 & orbit & date & day \\
\hline \hline ERS 1 & 8197 & $8-2-1993$ & $\mathrm{~d} 1$ \\
ERS 1 & 9700 & $24-5-1993$ & $\mathrm{~d} 2$ \\
ERS 1 & 19563 & $12-4-1995$ & $\mathrm{~d} 3$ \\
ERS 1 & 21066 & $26-7-1995$ & $\mathrm{~d} 4$ \\
ERS 2 & 1393 & $27-7-1995$ & $\mathrm{~d} 4$ \\
ERS 1 & 21567 & $30-8-1995$ & $\mathrm{~d} 5$ \\
ERS 2 & 1894 & $31-8-1995$ & $\mathrm{~d} 5$ \\
ERS 2 & 2896 & $9-11-1995$ & $\mathrm{~d} 6$ \\
ERS 2 & 6904 & $15-8-1996$ & $\mathrm{~d} 7$ \\
ERS 2 & 8908 & $2-1-1997$ & $\mathrm{~d} 8$ \\
ERS 2 & 9409 & $6-2-1997$ & $\mathrm{~d} 9$ \\
ERS 1 & 30585 & $21-5-1997$ & $\mathrm{~d} 10$ \\
ERS 2 & 10912 & $22-5-1997$ & $\mathrm{~d} 10$ \\
ERS 1 & 31587 & $30-7-1997$ & $\mathrm{~d} 11$ \\
ERS 2 & 11914 & $31-7-1997$ & $\mathrm{~d} 11$ \\
ERS 2 & 13417 & $13-11-1997$ & $\mathrm{~d} 12$ \\
ERS 2 & 13918 & $18-12-1997$ & $\mathrm{~d} 13$ \\
ERS 2 & 17926 & $24-9-1998$ & $\mathrm{~d} 14$ \\
ERS 1 & 40605 & $21-4-1999$ & $\mathrm{~d} 15$ \\
ERS 2 & 20932 & $22-4-1999$ & $\mathrm{~d} 15$ \\
\hline
\end{tabular}

represents the temporal extension of the 43 interferometric deformation maps generated and is actually the graphical representation of the system matrix $A$. The topographic component to be subtracted was estimated by using a digital elevation model from the Italian Military Geographic Institute ${ }^{1}$ with a spatial resolution of 1 arcsec in both latitude and longitude and a height accuracy of about 10-20 m. The zero-deformation phase value was taken as the mean value of the phases comprised within a square of size $0.004^{\circ}$ in a zone of no deformations.

The majority of the interferometric deformation maps resulted to be already consistent with respect to the closed-loop test. Only a couple of them turned out to be slightly biased, in most cases of only a few millimeters. Even for these small biases, however, correction resulted in a better consistence of all the deformation images with each other, as it was assessed at the end of the correction procedure by checking once again the histograms of all the possible closed loop combinations. The deformation sequence resulting from the least squares adjustment is shown in Fig. 4. The deformations are represented in centimeters and relate to the date corresponding to the first image acquired, i.e., February 8, $1993(d 1)$. The images have been masked for the sea in the lower part, while the sparse masked areas on the land are those where, due to the presence of decorrelation noise, the deformations could not be resolved. The deformations are consistent with the results from the GPS and leveling surveys performed periodically in the area [12], [14]. The subsidence, as seen along the radar line-of-sight, has a radial pattern centered on the east part of the city of Pozzuoli. Here the ground deformation is maximal, with a subsidence from February 1993 to April 1999, as measured from the interferometric data, of about $22.5 \mathrm{~cm}$ in the slant range direction, with a standard deviation estimated at the level of $0.4 \mathrm{~cm}$. The

${ }^{1}$ Courtesy of IREA-CNR.

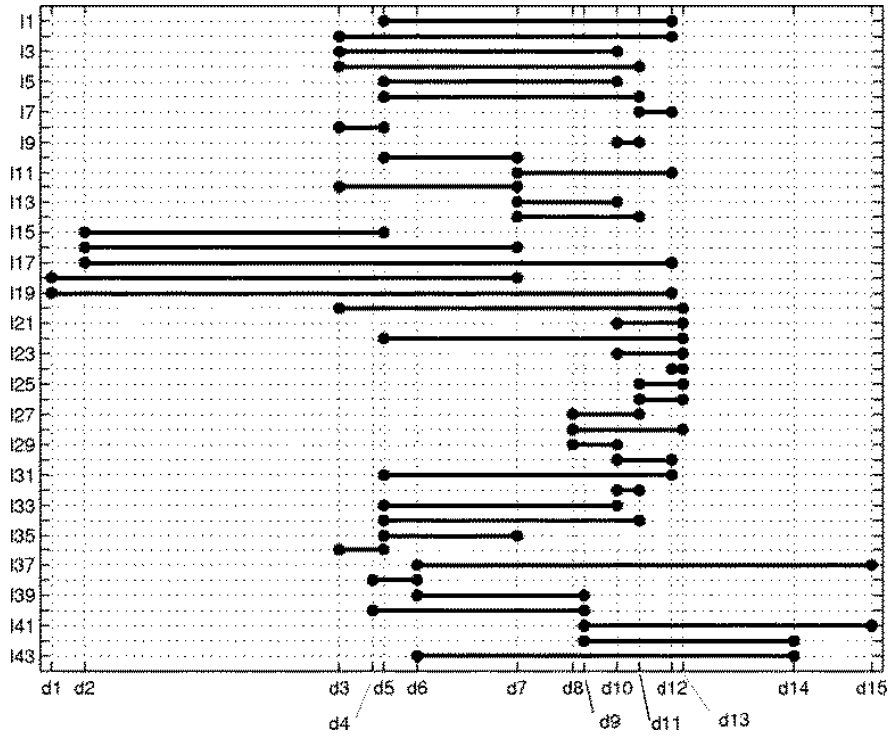

Fig. 3. Set of interferograms generated from the ERS dataset of Table I and used for the LS adjustment. The time spans are depicted as horizontal bars on a linear scale.

mean subsidence rate is of the order of about $0.3 \mathrm{~cm} / \mathrm{month}$, quite in agreement with the expected value from leveling data of $0.4 \mathrm{~cm} /$ month, although variations in the deformation rate are detected. Fig. 5 shows the values measured in the LS solution of Fig. 4 at the maximum subsidence point, which corresponds also to the region of maximum deformation according to the leveling results [14]. The error bars depicted in Fig. 5 represent the standard deviation computed, for each point, on a $6 \times 11$ window centered on the maximum deformation point. The addition of more interferograms and the computation of the solution at three additional days give a solution that seems to confirm the results of [9]. While the period June 1993-March 1995 remains still uncovered by the InSAR data, considerable variations in the subsidence rate apparently occur from 1995 to 1999 . The plot shows in fact three periods of relatively slow subsidence, in the first half of 1995 and of 1997, and from the end of 1998 to April 1999. In between these periods, subsidence seems to occur at an increased rate. The plot seems also to suggest that in January 1997 the deformation even changed its sign: however, the size of the error bars adds a considerable uncertainty to this results. Indeed, the very slow subsidence measured with leveling in this period also fits within these error bars. Notice that the set of interferograms $I 37, \ldots, I 43$, which are a combination of the SAR images corresponding to the set of days $(d 4, d 6, d 9, d 14, d 15)$, was added to the first 36 interferograms by interpolation. This was necessary because the presence of extremely large baselines did not allow to form interferograms by combining one image from a day of the set given above with one corresponding to the remaining days. In other words, the two sets of interferograms are disjoint. As already explained in Section III-B, in order to be able to obtain a unique solution a "simulated" interferogram has to be generated that connects the two sets. The preliminary solution obtained by adjustment of the first block was, therefore, used to generate the simulated interferometric deformations between 

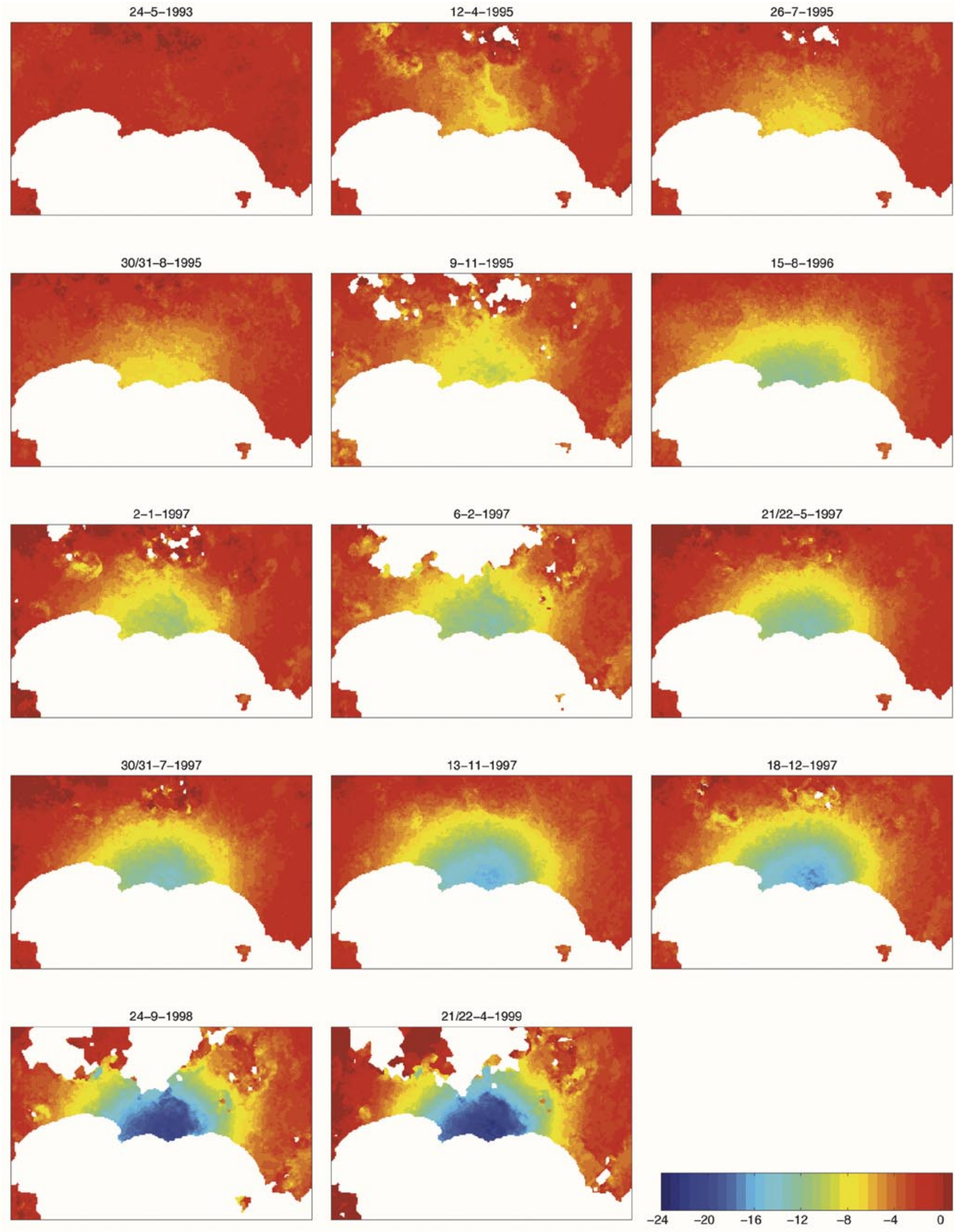

Fig. 4. Resulting deformations (in centimeters) at the 14 considered dates with respect to February 8, 1993. 


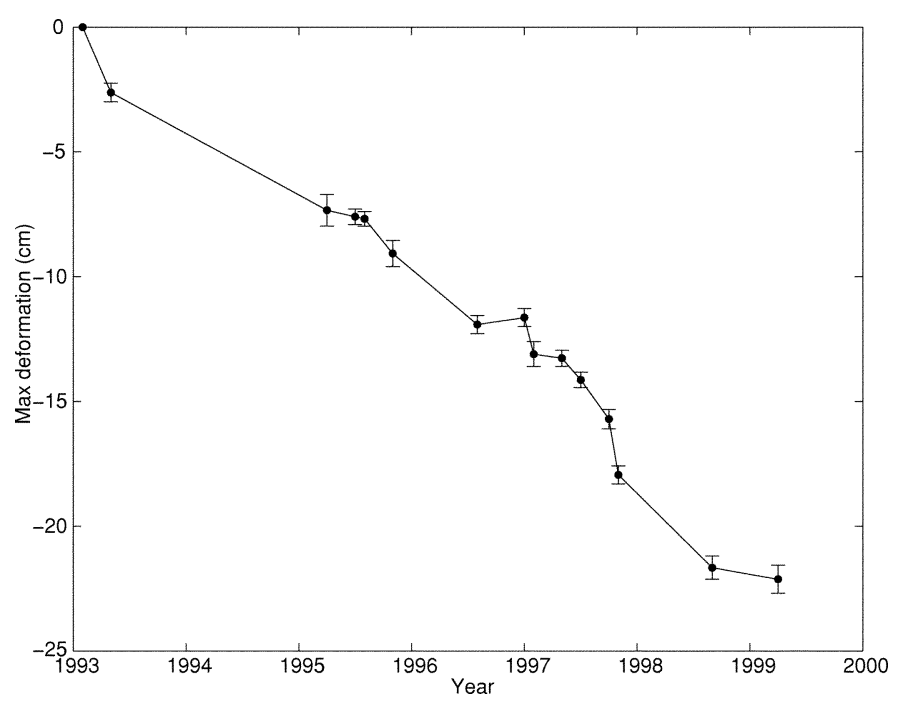

Fig. 5. Maximum subsidence values as measured from the solution of Fig. 4. The standard deviation values in the plot are computed on a $6 \times 11$ window centered on the maximum deformation point.

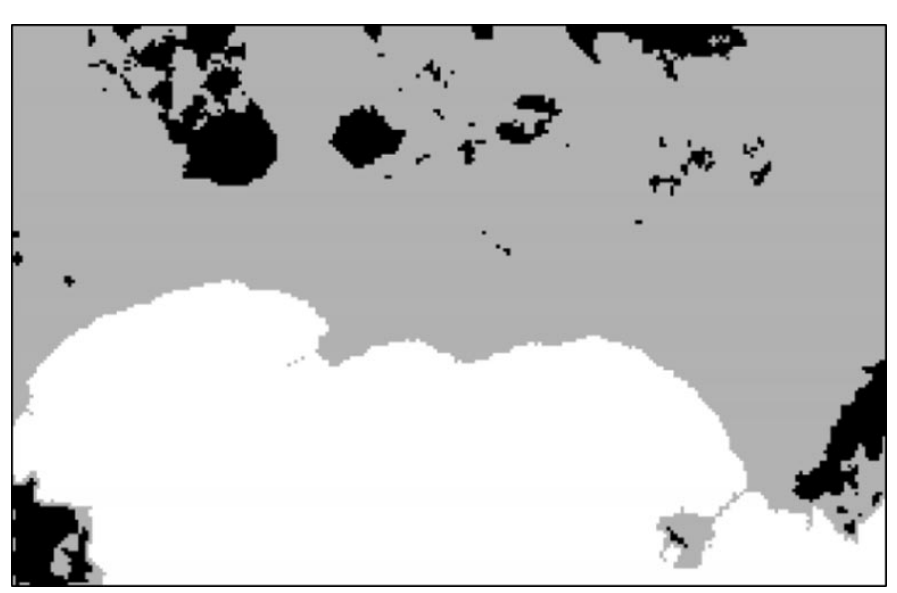

Fig. 6. Location of the pixels (depicted in black) that failed the overall model test.

days $d 1$ and $d 4$ (February 8, 1993 and July 26, 1995). The displacement map (which could be added as $I 44$ to the scheme of Fig. 3) was computed with linear interpolation of the solutions obtained at days $d 3$ and $d 5$. Of course, linear interpolation should be avoided as much as possible in a case like that of the Phlegrean Fields, whose deformation history teaches us that variations and even sudden inversions in the ground movements can always occur. The interpolation has, however, been applied on a four-month interval on a period, i.e., from April to August 1995, when no particular events were registered and subsidence seemed to proceed at a constant rate [14]. Even if a linear approximation has been used, therefore, for these reasons it can still be considered a reasonable assumption. Fig. 6 shows the position of the pixels that did not pass the overall model test. The majority of them are located in correspondence of topographic features, like the craters to the north of Pozzuoli, in the upper central part of the image, or the Posillipo hills, at its lower right side (see also the topography as shown in the intensity image of Fig. 2). This suggests the presence of a signal due to residual topography.

\section{DISCUSSION}

The LS method has several advantages with respect to retrieving the deformations by analyzing each interferogram singularly. The use of a database of interferograms permits a cross-check on the consistence of their deformations results with respect to biases, as shown with the so-called "closed-loop" test. Solving all the deformations as a unique least squares problem provides then a chronologically ordered sequence, i.e., a picture of the development of the deformation pattern in time, even if no direct interferograms between subsequent dates are available, either because the area has not been imaged or because the baseline is too large. Moreover, by applying the least squares, it is possible to reduce the effects of interferometric processing errors, which can be considered different (thus independent) in each interferogram. Notice that the same is not true for image-related errors, like for example atmospheric disturbances. If one image carries such an error, in fact, this will show up in two of the interferograms in (1) with different sign and will therefore be canceled out. The closed-loop test is thus not sensitive to such an error.

If the LS method on one hand does not provide a method to detect or subtract atmospheric artifacts, on the other hand because it merges several interferograms it allows to average them out at least partially. In fact, because atmospheric artifacts are image-related, it is reasonable to assume that interferograms computed from two tandem pairs, although spanning practically the same interval, are totally independent. Therefore, the proposed approach has also in this respect an additional advantage with respect to considering isolated interferograms. Of course, algorithms to detect atmospheric artifacts can always be added, as for example was done in [15] by using a method derived from the Permanent Scatterers approach [16], [17]. The same is true for topographic errors, which the testing procedure presented in this paper certainly helps to detect.

The fact that the proposed implementation of the LS procedure requires coregistration of all the images on the same master might be seen as a limitation of this approach. This is, however, not such a strict constraint, as the experience with ERS data suggests that up to baselines of about $600 \mathrm{~m}$ a good coregistration is possible. The coregistered image cannot be used in combination with the master, but it will form short baseline interferograms with other slaves. Moreover, as already stressed above, image coregistration on a common master is not strictly necessary for the implementation of this approach: the LS procedure can in fact be applied also on interferograms geocoded as usual on the desired reference system.

The presented approach is very similar to the singular value decomposition (SVD) approach of [15] and [18]. The method applies SVD on the same problem, reformulated however in terms of deformation mean velocities instead of deformation values (see (14) in the Appendix). This allows to connect disjoint subsets of interferometric maps without the need of the linear interpolation procedure explained in Section III-B.

In principle, SVD is more effective than least squares because it offers a way to find an approximate mathematical solution to a singular problem. Its "best" solution is, in fact, the minimum norm projection in the row space of the problem matrix. 
By formulating a problem with SVD, however, one poses certain implicit conditions, which are expressed by a number of equations equal to the dimension of the null space of the undetermined problem. Unfortunately these equations, although they allow to solve the problem correctly from the mathematical point of view, might express conditions that are unreasonable or even unacceptable from the physical point of view.

This is indeed what happens in the interferometric case when SVD is applied to minimize the norm of the mean velocities. As it is shown in the Appendix, this can give oscillations in the estimated mean velocities which have no physical justification.

In conclusion, SVD is certainly the "natural extension" of a Least Squares problem when this is underdetermined, but it might be necessary to radically change the formulation of the problem itself in order to have a physically sound solution.

\section{CONCLUSION}

A least squares approach for differential InSAR applications has been presented. With this approach, the least squares inversion of a database of interferometric deformation images on short- and long-term interferograms allows to generate a chronologically ordered sequence describing the evolution of the deformations in the course of the years. Moreover, the redundancy of the information contained in interferograms acquired on (partially) overlapping time intervals is used to filter out interferogram-dependent errors and to fill gaps due to temporal decorrelation. In this sense, the LS approach gives considerably better results than the use of single interferograms. The introduction of statistical testings allows control and quality assessment of the solution: application of a simple overall model test allowed in the presented case to identify systematic effects due to residual topography, but in successive implementations it could be used also for data snooping.

The LS approach was applied to study the terrain displacements in the Phlegrean Fields volcanic area in the period 1993-1999. The results, in good agreement with the expected deformations from other kind of data, clearly demonstrate the potentiality of such an InSAR database approach for geophysical studies and hazard monitoring.

\section{APPENDIX SVD FORMULATION}

Let us consider six consecutive acquisition dates $\left[t_{0}, \ldots, t_{5}\right]$, and suppose that interferograms are available on the intervals $\left(t_{0} t_{1}\right),\left(t_{1} t_{3}\right),\left(t_{2} t_{4}\right)$, and $\left(t_{4} t_{5}\right)$. The corresponding measured phase differences are respectively $\phi_{01}, \phi_{13}, \phi_{24}$, and $\phi_{45}$ (see Fig 7). According to [15, Eq. (15)], the SVD problem is then formulated as follows:

$$
\left\{\begin{array}{l}
\frac{\phi_{01}}{t_{1}-t_{0}}=v_{0} \\
\frac{\phi_{13}}{t_{3}-t_{1}}=v_{1} \frac{t_{2}-t_{1}}{t_{3}-t_{1}}+v_{2} \frac{t_{3}-t_{2}}{t_{3}-t_{1}} \\
\frac{\phi_{24}}{t_{4}-t_{2}}=v_{3} \frac{t_{3}-t_{2}}{t_{4}-t_{2}}+v_{2} \frac{t_{4}-t_{3}}{t_{4}-t_{2}} \\
\frac{\phi_{45}}{t_{5}-t_{4}}=v_{4}
\end{array}\right.
$$

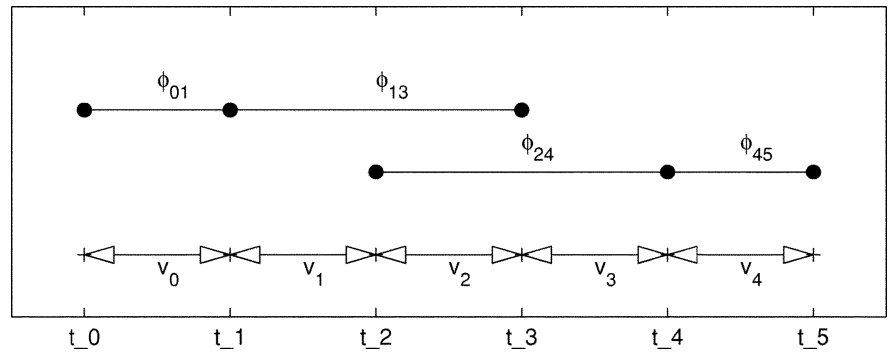

Fig. 7. Two disjoint sets of interferograms used for the example.

with $v_{i}(i=0, \ldots, 4)$ the unknown mean velocities, also represented in Fig. 7

Notice that the formulation of the problem in terms of the deformation velocities is equivalent to the formulation in terms of the deformation values except for a scale factor, namely the temporal interval over which each deformation is considered. In the nonsingular case, therefore, applying the SVD approach on the deformation rates, or the LS approach on the deformations, leads to identical results.

The system of equations corresponding to (13) is of the form $A * v=\phi / \Delta t$

$$
\left[\begin{array}{ccccc}
1 & 0 & 0 & 0 & 0 \\
0 & \frac{t_{2}-t_{1}}{t_{3}-t_{1}} & \frac{t_{3}-t_{2}}{t_{3}-t_{1}} & 0 & 0 \\
0 & 0 & \frac{t_{3}-t_{2}}{t_{4}-t_{2}} & \frac{t_{4}-t_{3}}{t_{4}-t_{2}} & 0 \\
0 & 0 & 0 & 0 & 1
\end{array}\right]\left[\begin{array}{l}
v_{0} \\
v_{1} \\
v_{2} \\
v_{3} \\
v_{4}
\end{array}\right]\left[\begin{array}{c}
\frac{\phi_{01}}{t_{1}-t_{0}} \\
\frac{\phi_{13}}{t_{3}-t_{1}} \\
\frac{\phi_{24}}{t_{4}-t_{2}} \\
\frac{\phi_{45}}{t_{5}-t_{4}}
\end{array}\right]
$$

The application of SVD to this underdetermined problem implies the assumption of an implicit condition, whose corresponding equation can be determined by imposing the orthogonality condition [19]: $A * b=0$, with $b$ the vector of the equation coefficients. This leads to the following solution:

$$
b_{0}=0 \quad b_{1}=\frac{t_{4}-t_{3}}{t_{2}-t_{1}} b_{3} \quad b_{2}=-\frac{t_{4}-t_{3}}{t_{3}-t_{2}} b_{3} \quad b_{4}=0 .
$$

Posing $b_{3}=1$, the implicit condition assumed to solve the problem is thus

$$
\frac{t_{4}-t_{3}}{t_{2}-t_{1}} v_{1}-\frac{t_{4}-t_{3}}{t_{3}-t_{2}} v_{2}+v_{3}=0
$$

Equation (16) can lead to odd results. Let us make the very simple assumption of a constant mean deformation velocity, which we can assume to be unitary, measured on time intervals of equal length

$$
\begin{aligned}
t_{2}-t_{1} & =t_{3}-t_{2}=t_{4}-t_{3} \\
\frac{\phi_{01}}{t_{1}-t_{0}} & =\frac{\phi_{13}}{t_{3}-t_{1}}=\frac{\phi_{24}}{t_{4}-t_{2}}=\frac{\phi_{45}}{t_{5}-t_{4}}=1 .
\end{aligned}
$$

The problem of (14) in this case takes the form

$$
\left[\begin{array}{ccccc}
1 & 0 & 0 & 0 & 0 \\
0 & \frac{1}{2} & \frac{1}{2} & 0 & 0 \\
0 & 0 & \frac{1}{2} & \frac{1}{2} & 0 \\
0 & 0 & 0 & 0 & 1 \\
0 & 1 & -1 & 1 & 0
\end{array}\right]\left[\begin{array}{l}
v_{0} \\
v_{1} \\
v_{2} \\
v_{3} \\
v_{4}
\end{array}\right]=\left[\begin{array}{l}
1 \\
1 \\
1 \\
1 \\
0
\end{array}\right]
$$




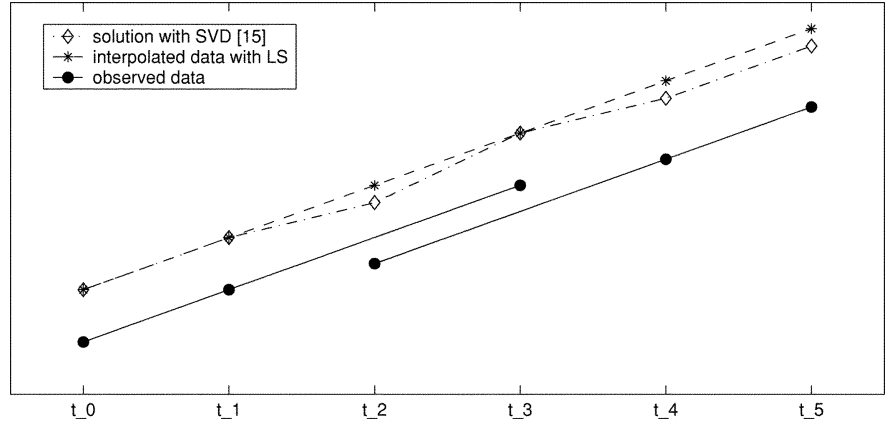

Fig. 8. Comparison of the solutions obtained by applying the LS and SVD approaches to the proposed example, in the case of a linear constant mean deformation velocity and equal time intervals. Notice that the shift in the $y$ axis (which represents the amount of deformation) between observed data and solutions has no significance, as it is applied only for visualization purposes.

where also the "missing" equation (16) has been added. Solving the system of (19) gives the following vector of mean velocities:

$$
v=\left[\begin{array}{lllll}
1 & \frac{2}{3} & \frac{4}{3} & \frac{2}{3} & 1
\end{array}\right]^{t} .
$$

This solution is illustrated in Fig. 8, where the original problem and the two examined solutions are shifted with respect to each other along the $y$ axis, which indicates the amount of deformation, only for the purpose of a better visualization (the $y$ axis has thus no scale). The picture represents the observed deformations (continuous lines in the low part of the figure) in the specific case considered, i.e., of a constant deformation velocity. It is important to remark here that only the deformation values between two instants, e.g., $t_{0}$ and $t_{1}$, or $t_{1}$ and $t_{3}$, are known. The mean velocities are computed by dividing the phase differences by the time interval [e.g., $\phi_{01} /\left(t_{1}-t_{0}\right)$ ]. According to the LS method presented in this paper, the two disjoint sets of interferograms are connected by interpolation, which in terms of the representation of Fig. 8 corresponds to shifting, for example, the value in $t_{2}$ to match the line between $t_{1}$ and $t_{3}$. The SVD approach of [15] brings to a different solution, also represented, in the simplest case, by (20) and in Fig. 8, which is affected by oscillations generated by the mathematical formulation of the problem. As for the reason of these oscillations, a first analysis suggests, as the probable cause, the fact that the chronological order of the velocities is not taken into account in the solution of the problem.

\section{ACKNOWLEDGMENT}

The author would like to thank E. Sansosti, G. Fornaro, R. Lanari, and P. Berardino of IREA-CNR for the interesting discussions and S. Borgstrom and G. P. Ricciardi of the Osservatorio Vesuviano for providing the information about the ground deformations at the Phlegrean Fields area. Many thanks go also to the two anonymous reviewers for their very helpful suggestions.

\section{REFERENCES}

[1] A. K. Gabriel, R. M. Goldstein, and H. A. Zebker, "Mapping small elevation changes over large areas: Differential radar interferometry," $J$. Geophys. Res., vol. 94, no. B7, pp. 9183-9191, July 1989.
[2] D. Massonnet and K. L. Feigl, "Radar interferometry and its application to changes in the earth's surface," Rev. Geophys., vol. 36, no. 4, pp. 441-500, Nov. 1998.

[3] H. A. Zebker, P. A. Rosen, R. M. Goldstein, A. Gabriel, and C. L. Werner, "On the derivation of coseismic displacement fields using differential radar interferometry: The Landers earthquake," J. Geophys. Res., vol. 99, no. B10, pp. 19617-19634, Oct. 1994.

[4] D. Massonnet, P. Briole, and A. Arnaud, "Deflation of Mount Etna monitored by spaceborne radar interferometry," Nature, vol. 375, pp. 567-570, June 15, 1995.

[5] H. A. Zebker and J. Villasenor, "Decorrelation in interferometric radar echoes," IEEE Trans. Geosci. Remote Sensing, vol. 30, pp. 950-959, Sept. 1992.

[6] R. Bamler and D. Just, "Phase statistics and decorrelation in sar interferograms," in Proc. IGARSS, Tokyo, Japan, 1993, pp. 980-984.

[7] S. Usai, "A new approach for long term monitoring of deformations by differential SAR interferometry," Ph.D. thesis, Delft Univ. of Technol., Delft, The Netherlands, 2001.

[8] S. Usai, E. Sansosti, P. Berardino, R. Lanari, G. Fornaro, M. Tesauro, and P. Lundgren, "Modeling terrain deformations at the Phlegrean Fields with InSAR," in Proc. IGARSS, Honolulu, HI, 2000, pp. 2245-2247.

[9] P. Lundgren, S. Usai, E. Sansosti, R. Lanari, M. Tesauro, G. Fornaro, and P. Berardino, "Modeling surface deformation observed with SAR interferometry at Campi Flegrei caldera," J. Geophys. Res., vol. 106, no. B9, pp. 19355-19367, Sept. 2001.

[10] C. C. J. M. Tiberius and F. Kenselaar, "Estimation of the stochastic model for GPS code and phase observables," Surv. Rev., vol. 35, no. 277, pp. 441-454, 2000.

[11] P. J. G. Teunissen, Adjustment Theory: An Introduction, ser. Mathematical Geodesy and Positioning. Delft, The Netherlands: Delft Univ. Press, 2000.

[12] G. Orsi, L. Civetta, C. Del Gaudio, S. deVita, M. A. Di Vito, R. Isaia, S. M. Petrazzoli, G. P. Ricciardi, and C. Ricco, "Short-term ground deformations and seismicity in the resurgent Campi Flegrei caldera (Italy): An example of active block-resurgence in a densely populated area," $J$. Volcanol. Geotherm. Res., vol. 91, pp. 415-451, 1999.

[13] G. Saccorotti, F. Bianco, M. Castellano, and F. Del Pezzo, "The July-August 2000 seismic swarms at Campi Flegrei volcanic complex, Italy," Geophys. Res. Lett., vol. 28, no. 13, pp. 2525-2528, July 2001.

[14] Osservatorio Vesuviano, "Int. Rep. on Surveying Activities,", 2001.

[15] P. Berardino, G. Fornaro, R. Lanari, and E. Sansosti, "A new algorithm for surface deformation monitoring based on the combination of small baseline DIFSAR interferograms," IEEE Trans. Geosci. Remote Sensing, vol. 40, pp. 2375-2383, Nov. 2002.

[16] A. Ferretti, C. Prati, and F. Rocca, "Nonlinear subsidence rate estimation using permanent scatterers in differential sar interferometry," IEEE Trans. Geosci. Remote Sensing, vol. 38, pp. 2202-2212, Sept. 2000.

[17] A. Ferretti, C. Prati, and F. Rocca, "Permanent scatterers in SAR interferometry," IEEE Trans. Geosci. Remote Sensing, vol. 39, pp. 8-20, Jan. 2001.

[18] G. Fornaro and E. Sansosti, "Private communication," unpublished.

[19] G. Strang, Linear Algebra and Its Applications. San Diego, CA: Harcourt Brace Jovanovich, 1988.

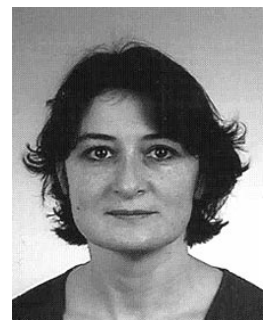

Stefania Usai received the M.S. degree in physics from the University of Pavia, Pavia, Italy, in 1993, and the Ph.D. degree in engineering from the Delft University of Technology, Delft, The Netherlands, in 2001.

She is currently a Researcher within the Delft Institute for Earth-Oriented Space Research and is a member of the Faculty of Aerospace Engineering, Delft University of Technology. She was a Research Fellow at the Brera Astronomical Observatory, Milan, Italy, in 1994, and subsequently in 1995 at the Politecnico of Milan, Milan, Italy. Her research interest is SAR interferometry, with particular emphasis on its application for geophysical studies. Her main interests are currently the development of algorithms for the optimal processing of interferometric datasets, and the fusion of Insar data with other kinds of geodetic and geophysical measurements for the purpose of deformation modeling and monitoring. 